Original Research Paper

\title{
Comparative Study of Two Reactive Routing Protocols with Realistic Mobility Model
}

\author{
John Tengviel and Agyemang Sylvester \\ Department of Computer Science, Sunyani Technical University, Sunyani, Ghana
}

\author{
Article history \\ Received: 08-10-2020 \\ Revised: 14-11-2020 \\ Accepted: 21-12-2020 \\ Corresponding Author: \\ John Tengviel \\ Department of Computer \\ Science, Sunyani Technical \\ University, Sunyani, Ghana \\ Email: john2001gh@yahoo.com
}

\begin{abstract}
A Mobile Ad-hoc Networks (MANETs) are dynamic wireless networks that can be formed without the need for any existing infrastructure in which each node can act as a host as well as a router. One of the main challenges of MANETs is the design of robust routing protocols that adapt to the frequent and randomly changing network topology. A variety of routing protocols have been proposed and several of them have been extensively simulated or implemented. In this study, we compare and evaluate the performance of two reactive routing protocols namely: The Ad-hoc OnDemand distance Vector (AODV) routing protocol, which is unipath and Adhoc On-Demand Multipath Distance Vector (AOMDV) routing protocol. We observed that the results of AODV significantly outperformed that of the AOMDV in most cases contrary to the report that AOMDV outperformed AODV, but concerning in the literature that the selection of the mobility model has a significant impact the performance of the routing protocol.
\end{abstract}

Keywords: AODV, AOMDV Routing Protocols, MANETs, SMOOTH Model, Performance Metrics

\section{Introduction and Motivation}

The development of wireless networks has brought about new prospects, e.g., Internet of Things (IoT), laptops and smartphones, for professional and private usage (Kocev et al., 2013). Mobile devices users are allowed to join and leave a network with no restrictions. In Mobile Ad hoc Networks (MANETs), there are no base stations, so each Mobile Node (MN) can act as a host as well as a router (Chlamtac et al., 2003) and can appear or disappear from the network at any time making the network dynamic. MANETs have several application areas including collaborative work (e.g., Conferences), emergency services (e.g., Earthquake, flood or storm, policing, firefighting) and military battlefields where networking setup may not exist or may be destroyed (Kumar and Mishra, 2012; Kumar and Kumar, 2015). Hence, MANETs have received a lot of attention from the research community (Ofosu et al., 2018a; 2018b).

Due to this feature of mobility, the network structure becomes unpredictable and changes frequently. One of the main challenges to be considered in the design of MANETs is, therefore, the development of an adaptive routing protocol that can efficiently and effectively find paths between communicating nodes. In order to find the appropriate routing protocol for a dynamic MANET's structure, the behavior of routing protocols needs to be examined with varying node speeds, the number of nodes and number of connections to make an informed decision of which routing protocol is the most effective. The above discussion leads us to believe that it is significant to initially understand and evaluate the performance of the two most prominent MANET reactive routing protocols namely: Ad-hoc On-Demand Distance Vector (AODV) and Ad-hoc On-Demand Multipath Distance Vector (AOMDV) routing protocols, using a most realistic mobility model. Results from this analysis can then be used to select the best base protocol for improvement.

This study studies two routing protocols with performance metrics in the NS2 simulator under a realistic mobility model. A mobility model simulates the movement of MNs and can be used to evaluate the performance of routing protocols in MANETs. We will compare the routing protocols using the following performance metrics:

- Packet Delivery Ratio (PDR): The rate of the data packets actually delivered to the destinations to those generated by the sources

- Normalised Load (NRL): The ratio of the total number of routing packets transmitted during 
simulation to the total number of packets actually received at the destination node

- Average End-to-End Delay (AED): The average time that packets take from the source node to reach the destination the packets received

- Throughput (THP): Measured in bps or kbps, is the number of bits received by the receiver from the various nodes over total simulation time

The remaining of this study is categorised as follows: Section 2 presents related work, section 3 describes routing protocols, including details of AODV and AOMDV and introduces the SMOOTH mobility model. Section 4 gives the simulator and implementation, section 5 shows results analysis and section 6 presents our conclusions and suggests possible future directions.

\section{Related Work}

Singh et al. (2017), the authors studied the performance of AOMDV under different mobility models and concluded that mobility models impact on the performance of routing protocols. A comparison also was made between AOMDV and AODV with varying models of mobility and found that AOMDV performed better than AODV in all mobility models. Parekh (2013), the authors evaluated the performance of AODV and AOMDV routing protocols in MANET. They concluded that AOMDV outperformed AODV routing protocol in terms of PDR, THP, AED and PDP with pause time and the number of connections. Moudgil and Rana (2017), the authors investigated the performance of single path routing protocol (AODV) and multipath routing protocol (AOMDV) under different mobility conditions. The authors reported that AOMDV protocol is more robust than AODV protocol due to its ability to find and use alternative paths in case of a path failure in high mobility MANETs. They, therefore, noted that in high speed mobility MANETs, AOMDV gives better performance than AODV as it has better PDR and less AED but more routing overhead. Prabhu and Krishan (2015), the authors studied the performance of two types of on-demand routing protocols: AODV and AOMDV with metrics such as PDR, THP, AED and Packets Dropped (PDP) with varying number of nodes. They observed that AOMDV is better than AODV in terms of PDR, PDP and AED. This is due to the ability of AOMDV to search alternative paths when a link is disconnected. AODV, however, indicated better performance than AOMDV concerning THP. Hence, the authors concluded that AOMDV is better on-demand routing protocol than AODV since it provides better statistics for PDR and PDP.

Ding et al. (2012), the authors analysed the performance routing of AODV protocol in MANETs and concluded that in a low-speed movement, the packet delivery rate of AODV is higher than AOMDV. Thus,
AODV is suitable for marine environment applications. They also indicated that the AODV routing protocol outperformed the DSDV in THP, fairness and stability. Biradar et al. (2010), the authors compared and evaluated the performance of two types of on-demand routing protocols - AODV and AOMDV based on PDR, NRL, AED and PDP. They noted that the AODV performed better than AOMDV in terms of routing overhead and packet delay whiles AOMDV outperformed AODV in terms of PDR and PDP with pause time. Parita and Pooja (2014), the authors represented a simulation-based comparison of two variants of a MANET routing protocol, i.e., AODV and AOMDV which was performed in NS2.35. The performance metrics measured are PDR, THP and AED with varying number of nodes. The authors concluded that AODV and AOMDV performed almost have the same PDR, although AOMDV performed lesser in terms of THP and AED as compared to AODV. They also observed that for applications where the delay is bound then AODV would be a better choice. Alslaim et al. (2014), the authors performed a comparative study on MANET routing protocols such as AODV, DSDV, DSR and AOMDV. The analysis was performed with PDR and AED varying with the number of nodes and maximum speed. The authors reported that AODV is the best of all four protocols in terms of PDR and DSDV having the least AED. They went further to propose an enhancement of AODV to reduce AED. Shafi and Abidi (2013) compare the performance of three on-demand routing protocols including; DSR, AODV and AOMDV with varying speed of nodes. The authors concluded that the AOMDV produced the best results in AED and THP. Also, they noted that the AODV gives the best performance in PDR and Routing Overhead (ROH).

\section{Routing Protocols}

MANETs are multichip wireless self-organising networks, where the nature of the network structure changes continuously. The MNs use the idea of forwarding packets through intermediate nodes for the source and destination nodes when outside their transmission range. Thus, all the nodes of MANETs act as routers and therefore participate in route discovery and maintenance for other nodes in the network. The categories of routing protocols are namely: Reactive or on-demand, global or proactive (table-driven) and hybrid protocols (Obiniyi and Olanrewaju, 2015; Patil, 2012).

\section{Reactive Routing Protocols}

In Reactive (On-demand) routing protocols, routes are established through route discovery when needed by the source node. The Dynamic Source Routing (DSR), AODV and Temporally Ordered Routing Algorithm (TORA) (Obiniyi and Olanrewaju, 2015; Patil, 2012) are examples of on-demand routing protocols. 


\section{Proactive Routing Protocols}

Proactive (Table-driven) routing protocols attempt to update route information to all destinations at regular intervals regardless of whether the routes are currently being used or not (Obiniyi and Olanrewaju, 2015; Patil, 2012). Examples of table-driven routing protocols are Optimised Link State Routing (OLSR), Destination Sequenced Distance Vector (DSDV) and Fisheye State Routing (FSE) (Obiniyi and Olanrewaju, 2015; Patil, 2012).

\section{Hybrid Routing Protocols}

Hybrid routing protocols integrate the features of both reactive and proactive routing protocols. They take the advantages of both the reactive and proactive routing protocols but may need an additional device such as GPS for the location of nodes, either separate or inbuilt into the communication device, to function (Obiniyi and Olanrewaju, 2015; Patil, 2012). An instance of a hybrid routing protocol is the Zone Routing Protocol (ZRP) (Obiniyi and Olanrewaju, 2015; Patil, 2012).

The reactive routing protocols are the most efficient when compared to the proactive and hybrid routing protocols. The reactive routing protocols tend to rely on simple broadcast techniques and local repair to establish or repair paths. However, the simple flooding approach for path discovery leads to a reduction of their efficiency and effectiveness as a routing protocol causing increasing routing delay and overhead with time. The AODV is selected for study because it is the most popular, best representative and most researched protocol in the research community (Sedrati et al., 2011; Vijayavani and Prema, 2012).

\section{Ad hoc On-Demand Distance Vector Routing}

Ad hoc On-demand Distance Vector (AODV) is a reactive routing protocol (Ofosu et al., 2018a; 2018b; Obiniyi and Olanrewaju, 2015; Patil, 2012; Patil et al., 2012). AODV is also called source-initiated reactive routing because routes are generated only at the time a sending node wishes to send data to a target destination. A path established between a source and destination is maintained until the source has no more data to send or the path becomes unavailable. The AODV is selected for use in this study because it is the most popular, best representative and most researched protocol in the research community (Sedrati et al., 2011; Vijayavani and Prema, 2012).

The route discovery mechanism of AODV is based on Route Request (RREQ), Route Reply (RREP) and Route Error (ERROR) messages. AODV uses the idea of simple flooding to forward RREQ packets from the source node to all other nodes in the network to discover routes (Obiniyi and Olanrewaju, 2015; Patil, 2012; Patil et al., 2012).
An RREQ is broadcast by the source node to locate the destination node when it has data to send. When each intermediate node receives an RREQ, a path to the source is established. If a receiving node is not the destination, has not previously received the RREQ and does not have a current route to the target, then it will broadcast the RREQ to all nodes within wireless range. The receiving node will only create an RREP if it is the destination node or it has a current path to the destination. The RREP is then unicast in a hop-by-hop fashion back to the source. As the RREP is transmitted back to the source, each intermediate node stores a path to the destination. When the source receives an RREP, it registers the path to the target and then the data transmission begins. In the case of receiving multiples RREPs, the source will select the path with the shortest hop count.

\section{Ad Hoc On-Demand Multipath Distance Vector Routing}

Ad-hoc On-demand Multipath Distance Vector (AOMDV) (Ofosu et al., 2018a; 2018b; Varshney and Maheshwari, 2016) Routing protocol is an extension to the AODV protocol with multiple paths. The routing records for each targeted node consists of a table of the next-hop together with the related hop counts. Every next hop has the same sequence number. This supports in keeping a record of a path. For an individual destination, a node maintains the advertised hop count, which specifies the highest hop count for all the routes, which is used for distributing path advertisements of the intended target. Each duplicate path advertisement received by a node specifies an alternative route to the target node.

A node avoids looping by accepting alternative paths to the destination if it has a smaller hop count than the listed hop count for the targeted node. Since the maximum hop count is used, the advertised hop count, therefore, does not change for the same sequence number. When a path advertisement is received for a destination with a higher sequence number, the next-hop list and the advertised hop count are reinitialised. The benefit of using AOMDV is that it allows intermediate nodes to reply to RREQs, while still selecting disjoint paths.

But, AOMDV has extra message overheads during path discovery due to increased flooding that is the broadcasting of messages to cover all the nodes within a network and because of its multipath nature, the target node replies to the several RREQs which results in longer delay and higher overhead. 4

\section{Mobility Models}

A mobility model is developed to imitate the movement pattern of MNs with regard to time. Mobility models can be used to evaluate the performance of mobile ad-hoc networks. The mobility model plays a 
significant role in determining the protocol performance (Pandey and Mishra, 2017). Thus, it is essential to study and analyse various mobility models and their effect on mobile ad Hoc Network protocols (Pandey and Mishra, 2017). Mobility models are mainly made up of two types, namely: Synthetic and trace-based (Camp et al., 2002; Tengviel and Diawuo, 2013; Tengviel et al., 2012; 2013). Synthetic models attempt to realistically represent the behaviours of MNs without the use of any real-world measurements, while traces are movement patterns observed in real life systems.

In this study, we use the trace-based SMOOTH (SMTH) mobility Model, which is both realistic and straightforward (Munjal et al., 2010; 2011). The SMTH mobility model generates traces that better match human movements than many other mobility models (Munjal et al., 2010; 2011). In SMTH, communities are represented as clusters of unequal sizes. This is done to imitate realistic scenarios, where popular communities are more likely to attract people and are therefore visited more frequently and for a longer duration. Human beings go to places where they have something to do and even within the same communities, some areas are visited more often than others. SMTH depicts this movement based on real-world data. The movement patterns that are developed by SMTH follow a power-law distribution and MNs' motions are created by a pre-set probability (Munjal et al., 2010; 2011).

\section{Methodology}

To compare the two on-demand ad-hoc routing protocols, it is best to use identical simulation environments for their performance evaluation.

\section{Simulating Advanced Mobility Model}

BonnMotion (2016) is a free Java-based software, which can be used to create and analyse mobility scenarios for the investigations of mobile ad hoc networks properties. BonnMotion supports several mobility models such as Manhattan Grid, Gauss Markov, Tactical Indoor, SLAW, Disaster Area and SMOOTH.

The latest version of the BonnMotion is v3.0.1 and it is compatible with the NS-2.35. BonnMotion-3.0.1 was released on 18th February 2016 by the Communication Systems Group at the University of Bonn Germany, the Tailors Group at the Colorado School of Mines, Golden, CO, USA and the Distributed Systems Group at the University of Osnabrück, Germany (BonnMotion, 2016).

The user may specify the choice of mobility model, number of nodes, the simulation duration, initial time to skip at the beginning of the simulation and simulation area. The movement file needs to be converted into the format of NS-2 since its structure is different from BonnMotion's.

\section{The Simulator and Implementation}

The simulation was carried out in Network Simulator version 2.35 (NS-2.35) which is a discrete event-driven network simulation tool (Kevin and Kannan, 2011; Soni et al., 2013). NS-2 is an opensource program which is suitable for developing new protocols, comparing different protocols, traffic evaluations and other performance testings. NS-2 supports several network mechanisms such as UDP, TCP, FTP, routing and mobility models.

The simulation was performed on an (Ubuntu-14.04, 2016) virtual machine (VirtualBox, 2016) under a Windows 7 host. Mobility scenarios and traffic patterns were generated for use in the simulation. Each simulation was run five times and the average values extracted.

Figure 1 explains the main steps of simulation used in this study, that was implemented by using NS-2, where the code was written in a Tcl language and the outputs of the NS-2 are trace file and NAM file. setdest and cbrgen.tcl are utilities that come with NS-2 which may be used to generate movement and traffic patterns.

The trace files were extracted using the AWK. The performance metrics graphs were drew using Matlab for analysis.

The flowchart in Fig. 1 explains the sequence of steps used for the simulation.

Step1: Start.

Step2: Generate the traffic generation file "CBR file" that generated by "cbrgen.tcl" file that supported by NS-2. this script found in(ns-allinone2.35/ns-2.35/ind_util/cmu_scen_gen/).

Step3: Create the movement file using BonnMotion. Then the movement file is converted into the format of NS-2 since its structure is different from BonnMotion's.

Step4: Then generate MANET's scenario (movement file) using the support of NS-2 by the "setdest" script.

Step5: Generate "tcl" script that represents the simulation environment of MANET with mobility model for one routing protocols.

Step6: Select suitable parameters that input to this "tcl" file in the NS-2 in order to perform the simulation and the outputs are "NAM" file or display and trace file contain all the simulation event to analysis.

Step7: Use AWK/Perl to extract the trace file

Step8: Analysis of the trace file and compute the Performance Metrics for the network (throughput, drop packets, end to end delay, packets delivery and normalise routing load).

Step9: Calculate the average of the performance metrics for each routing protocols file.

Step10: Then draw the results with a suitable parameter by using MATLAB.

Step11: End. 


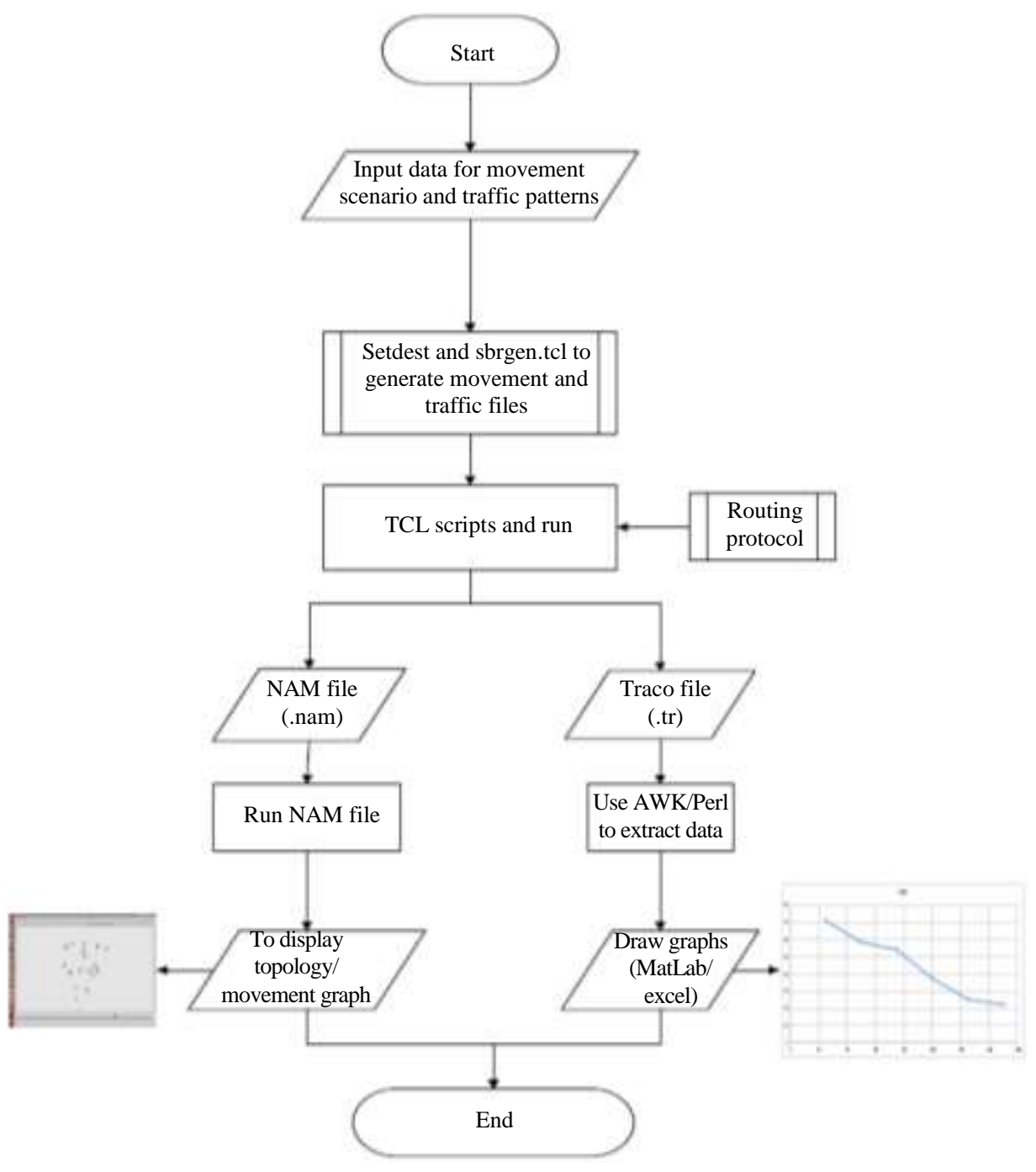

Fig. 1: The simulation steps

Table 1: Parameters for simulation

\begin{tabular}{ll}
\hline Parameter & Values \\
\hline Propagation model & Two Ray Ground Wave \\
Network interface type & Phy/WirelessPhy \\
MAC type & Mac/802.11 \\
Interface queue type & Queue/Drop Tail \\
No. of nodes & $20,25,75,100,125,150$ \\
Simulation time & $1200 \mathrm{sec}$ \\
Min. speeds & $1 \mathrm{~m} / \mathrm{sec}$ \\
Max. speeds & $5 \mathrm{~m} / \mathrm{sec}$ \\
Pause time & $1 \mathrm{~m} / \mathrm{sec}$ \\
Area & $1000 \times 1000 \mathrm{~m}$ \\
Connections & 5 \\
Mobility models & SMTH \\
\hline
\end{tabular}

\section{Mobility and Traffic Models}

This section describes experiments conducted with AODV and AOMDV under the SMTH model using three different scenarios.
The simulations were based on the parameters shown in Tables 1 and 2. The number of nodes was set to 150 as default to simulate a large-scale network (Chlamtac et al., 2003). A default maximum mobility speed of $5 \mathrm{~m} / \mathrm{s}$ was used to represent a high-speed cyclist or low-speed vehicle (Chen and Chang, 2003). A medium source-destination of 5 connections (i.e., traffic flows) was selected, each producing 4 data packets per second to simulate low traffic flow (Al Amri et al., 2010; Gupta et al., 2013).

The base values were then modified for each of the different scenarios as specified in the remaining subsections. The simulation was conducted in a network area of $1000 \times 1000 \mathrm{~m}$. Pause and simulation times were taken from the report (Munjal et al., 2011) and set to 1 and $1200 \mathrm{sec}$, respectively. The standard packet size of 512 bytes was used. User Datagram Protocol (UDP) was selected at the transport layer, allowing a message to be sent without an initial transmission setup path. 
Table 2: Parameters of SMOOTH mobility model

\begin{tabular}{ll}
\hline Parameters & Values \\
\hline Nodes & 150 \\
Area $\left(\mathrm{m}^{2}\right)$ & $1000 \times 1000$ \\
Clusters & 10 \\
$\left(\alpha, f_{\min }, f_{\max }\right)$ & $(2.2,5,500)$ \\
$\left(\beta, p_{\min }, p_{\max }\right)$ & $(2.1,10 \mathrm{~s}, 6 \mathrm{~h})$ \\
\hline
\end{tabular}

\section{Varying Number of Nodes}

In this scenario, the number of nodes varied from 20 , $25,50,75,100,125$ and 150, with all other parameters set to the values described in Table 1 .

\section{Varying Speed of Nodes}

In this scenario, the speeds of nodes were varied from 1 to $5 \mathrm{~m} / \mathrm{s}$ in steps of $1 \mathrm{~m} / \mathrm{s}$, with all other parameters set to the values described in Table 1 .

\section{Future Studies}

Firstly, to design an efficient routing protocol that can help to improve the route discovery phase. This routing protocol should:

- Allow only a specific set of nodes to rebroadcast the RREQ in cases where there is no available information in the routing table, to eliminate redundant RREQ packets

- The efficient routing protocol should include only those nodes in the end-to-end path which have a good level of residual energy, rather than establishing a route containing nodes which have low residual energy and will soon be unavailable due to their batteries running out

\section{Varying Number of Connections}

In this scenario, the number of connections was varied from 5 to 25 in steps of 5 , with all other parameters set to the values described in Table 1 .

\section{Results and Discussion}

In this section, we present results from the simulations described in the previous section.

\section{Effect of Varying Number of Nodes}

Figure 2 shows the PDR of AODV and AOMDV routing protocols with a varying number of nodes under the SMTH model. We can observe that the PDR of both routing protocols decreases with the increasing number of nodes except at 125 nodes where both routing protocols increased significantly but fall tremendously at 150 nodes. Though the PDR of AOMDV routing protocol increased tremendously at nodes 125 , it still falls short of the AODV routing. Thus, the AODV routing protocol performed better than AOMDV for the entire period with an increase in the number of nodes. AOMDV routing protocol shows lower PDR than AODV because AOMDV does not respond quickly to congestions and link failures through its multiple path discovery, maintenance and local search by the upstream node. In all cases, the PDR of AODV performed better than AOMDV under the SMTH model.

Figure 3 shows the throughput of AODV and AOMDV routing protocols with an increasing number of nodes under the SMTH mobility model. It can be observed that THP of both routing protocols decreases as the number of nodes increases except at 125 nodes where both increased significantly but fall again at 150 nodes. AODV routing protocol has better THP than AOMDV routing protocol because of an efficient path discovery and maintenance processes. The AODV routing protocol outperformed the AOMDV throughout the period in which the number of nodes increases. Thus, the possibility of an increase in contention and collisions in AOMDV leads to an increase in path disconnections, which finally necessitating more path discoveries and increased path repairs resulting in lower THP.

Figure 4 shows an increase in NRL of AODV and AOMDV routing protocols with an increasing number of nodes. It can be presented that the AODV routing protocol outperformed AOMDV routing protocol with an increasing number of nodes. Though the NRL values of AODV routing protocol are increasing very minimally, the values of AOMDV are both increasing and much higher values. This is due to the used of multipath routing or multiple paths maintenance, whereas AODV uses single path discovery and maintenance. Hence, it can respond more rapidly to connection changes than AOMDV routing protocol reducing the number of routing load used. The NRL of AODV increases throughout the period with the number of nodes. The broadcasting of RREQ, RERR and RREP for path repair and discovery are reduced in AOD, resulting in a lower NRL. The NRL of AOMDV is higher and increases as the number of nodes increases because of rebroadcasting of control packets as a result of multiple paths discoveries and repairs.

Figure 5 shows the AED of AODV and AOMDV routing protocols with an increased number of nodes.

It can be noted that the AED of both routing protocols increases as the number of nodes increases except at 100 nodes where it falls and then rises again for both protocols. This is due to the congestion of nodes in the network causing link failures, leading to dropped packets and delays. Thus, when there is a disconnection along a path in the network, AOMDV attempts to find an alternative route from among the backup paths between the sending and receiving node pairs or a new path searching is begun when the path is not located in backup paths resulting in an additional delay to the packet delivery period. 
However, AOMDV may find an alternative path faster from the backup paths as the nodes become enormous due to increase in several accessible paths resulting in faster packet delivery, hence lower AED.

The AODV AED is higher than that of AOMDV because of use of unipath and the initiation of fresh path discovery or repairs whenever there are path disconnections.

\section{Effect of Varying Speed of Nodes}

Figure 6 demonstrates the PDR of AODV and AOMDV routing protocols with different nodes speeds. It can be seen that AODV achieves a higher PDR as compared to AOMDV protocol with the increasing speed of nodes. Both routing protocols have comparable PDR trend at low speed, but that of AOMDV starts to decay to $99.62 \%$ at speeds of $4 \mathrm{~m} / \mathrm{s}$ whereas that of AODV is steady in the same period. AODV likewise reduced to $99.98 \%$ whereas that of AOMDV remains stable at a speed of $5 \mathrm{~m} / \mathrm{s}$ with AODV declining to $99.97 \%$ but still being higher. This is due to path disconnections, which are based on the speed increases between nodes. Whenever the speed between any two nodes is higher, this can cause a possible path disconnection which may lead to a dropped packet, but when the speed increase leads to constant mobility between nodes may not result in link disconnections. AODV routing protocol can respond more quickly through local repair or route rediscovery by the source or intermediate nodes than AOMDV routing protocol which needs to select another path from multiple paths or initiate a fresh route discovery when a path is not available. Thus, making it's responded slow to the path failure leading to lower PDR.

Figure 7 presents the comparative analysis between AODV and AOMDV in terms of THP with the speed of nodes in the network. The THP in AODV routing protocol is higher at the initial speed of $1 \mathrm{~m} / \mathrm{s}$ than AOMDV routing protocol.

But the AOMDV routing protocol, however, outperformed the AODV at speeds of 2 to $3 \mathrm{~m} / \mathrm{s}$ because at lower speeds the paths disconnections are not frequent so finding an alternative path from the backup paths is quicker in AOMDV than in AODV. At higher speeds of 4 to $5 \mathrm{~m} / \mathrm{s}$, the AODV outperformed the AOMDV, since, with an increase in the speed of mobile nodes, the possibility of link failure is high. At a more top speed, the path disconnections are often in both routing protocols resulting in loss of packets. However, AODV through single path discovery process can rediscover or maintain routes with ease whereas in AOMDV there are multiple links from one node to another, so link disconnection may affect several nodes and paths making the initial routes discovered useless resulting in lower THP. Thus, in most cases, AODV outperformed AOMDV because of its single path discovery and repair.

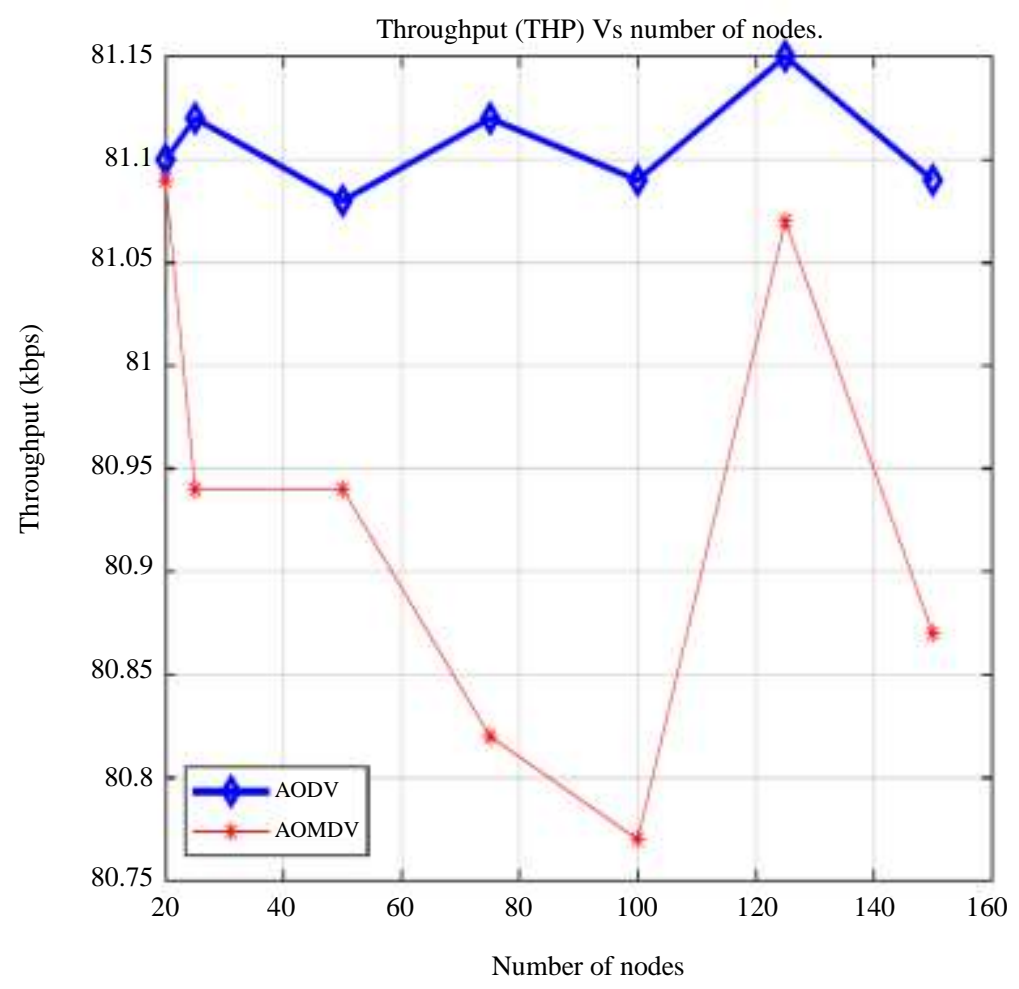

Fig. 2: Throughput with number of nodes 
John Tengviel and Agyemang Sylvester / American Journal of Engineering and Applied Sciences 2020, 13 (4): 803.818 DOI: 10.3844/ajeassp.2020.803.818

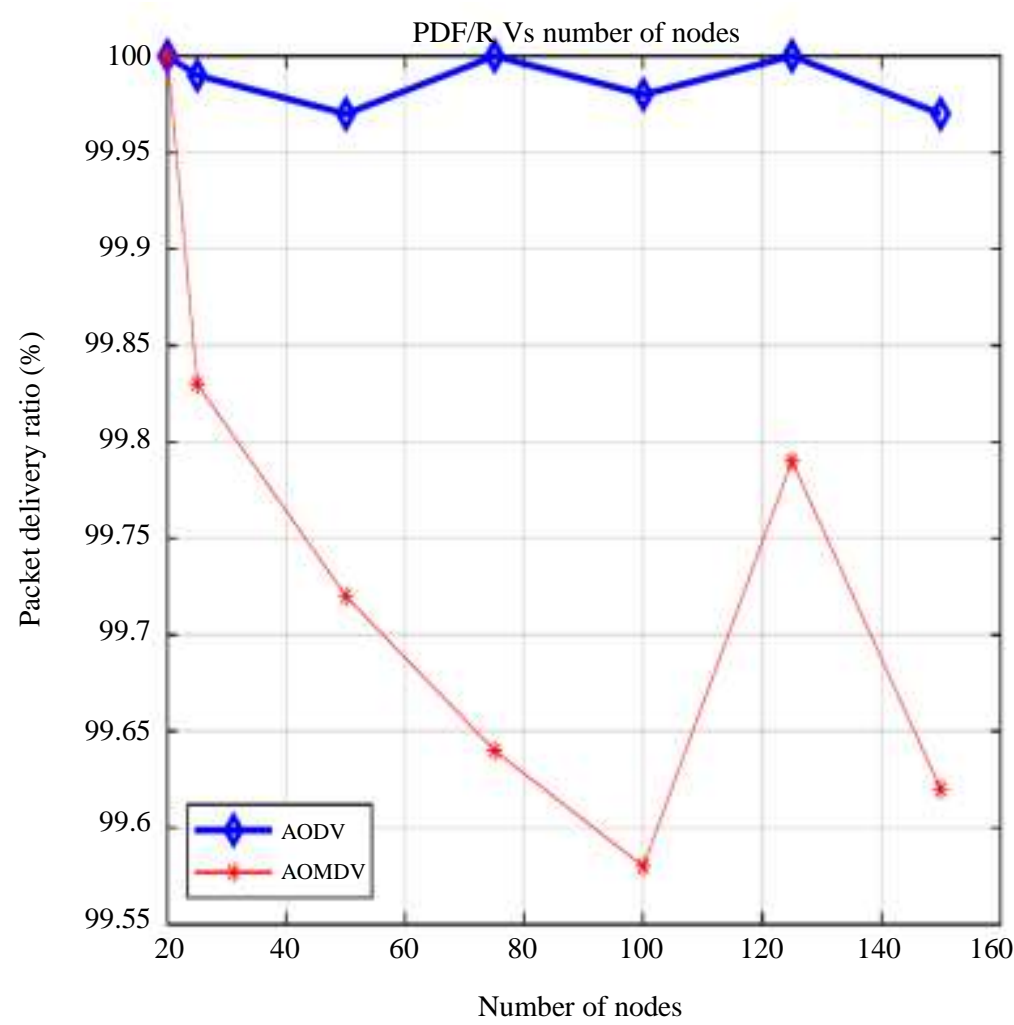

Fig. 3: Packet delivery ratio with number of nodes

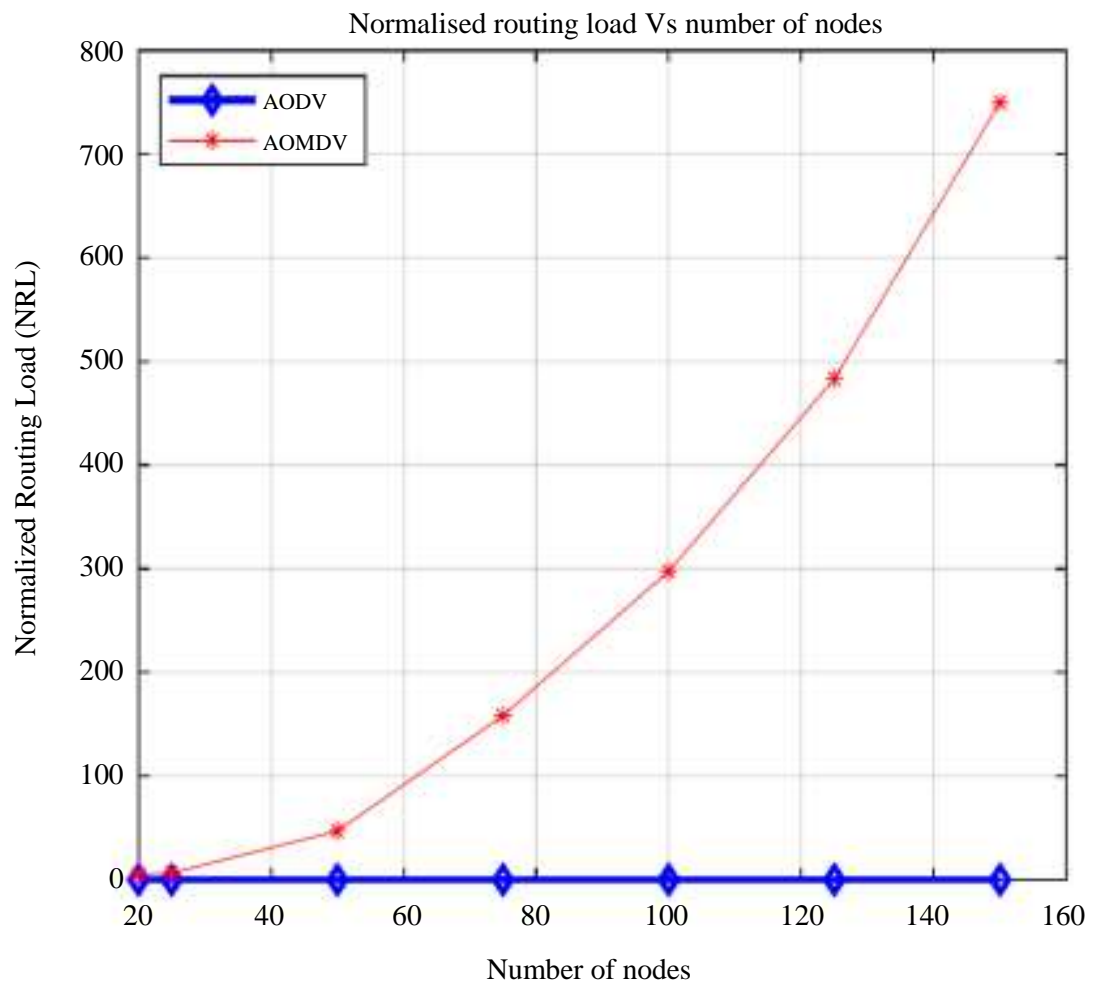

Fig. 4: Normalised routing load with number of nodes 
John Tengviel and Agyemang Sylvester / American Journal of Engineering and Applied Sciences 2020, 13 (4): 803.818 DOI: 10.3844/ajeassp.2020.803.818

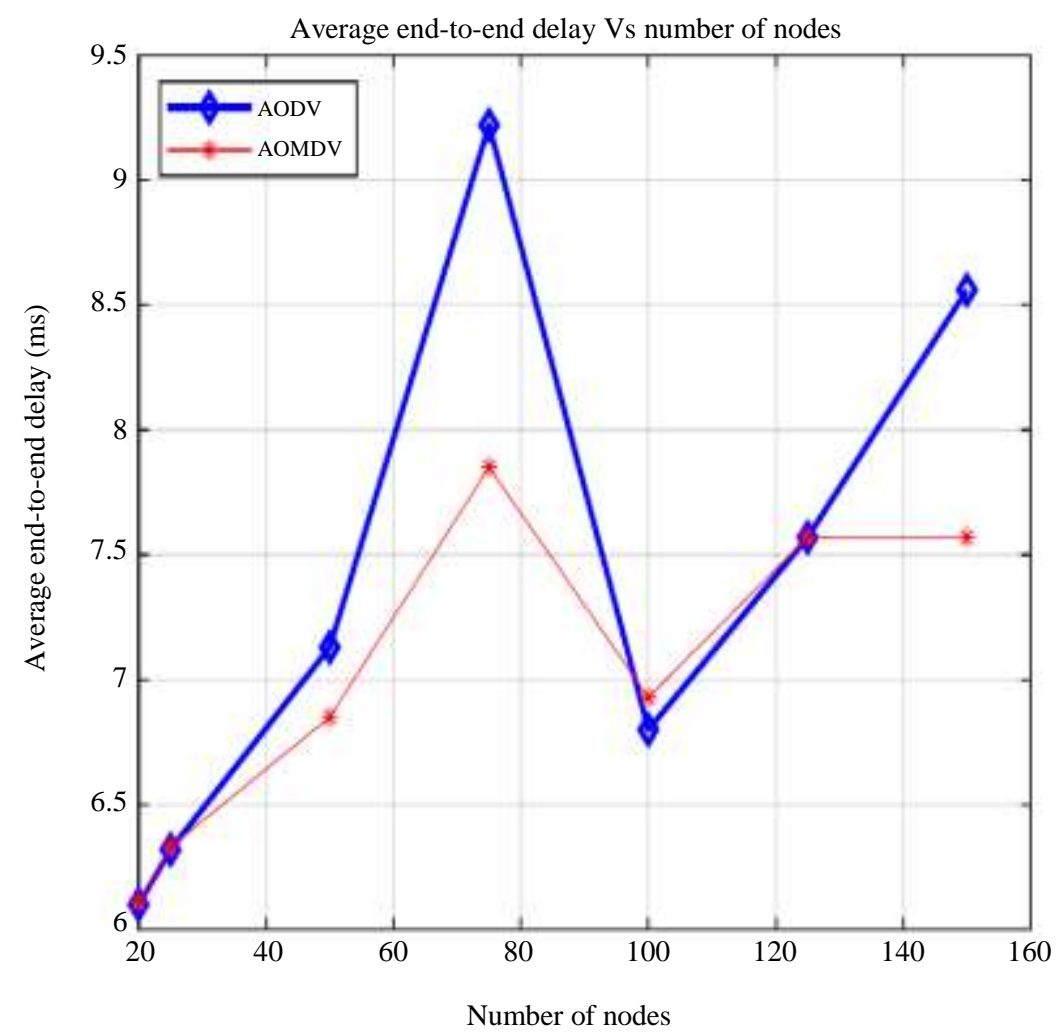

Fig. 5: Average end-to-end delay with number of nodes

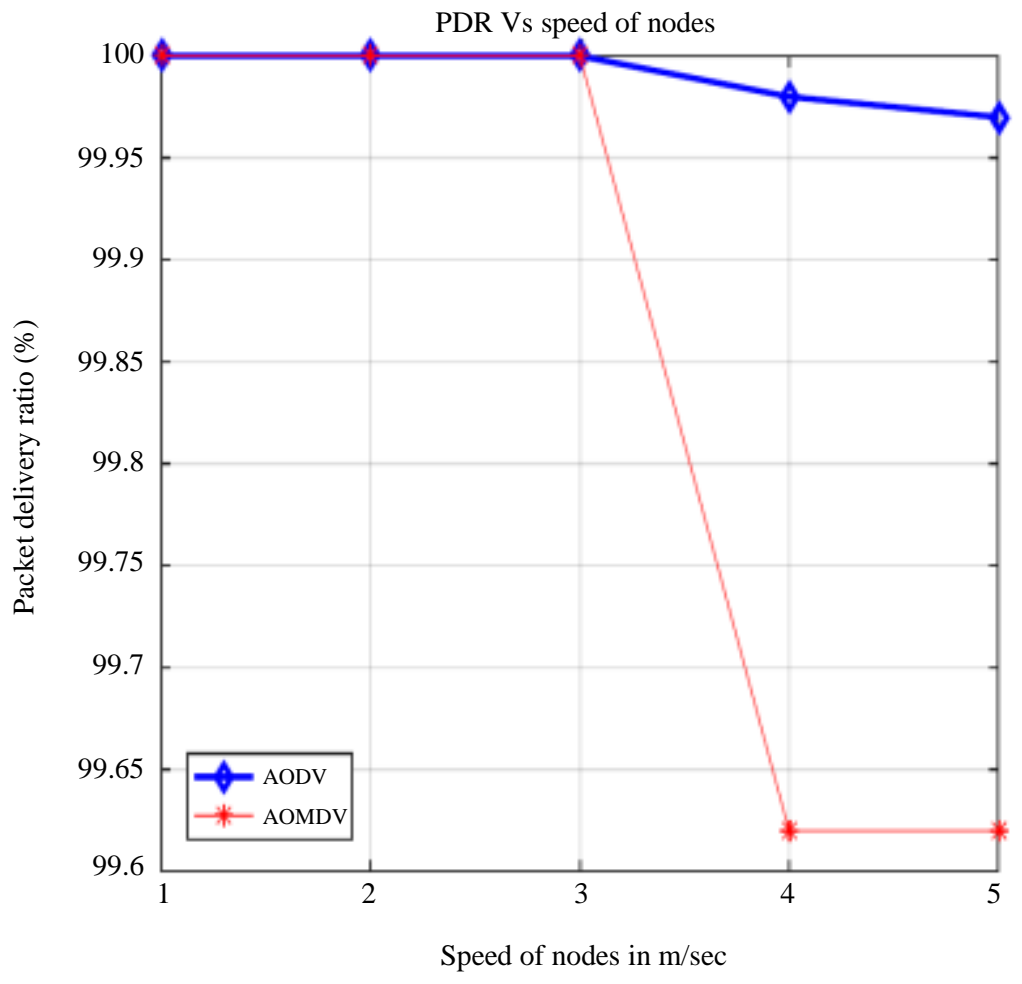

Fig. 6: Packet delivery ratio with speed of nodes 
John Tengviel and Agyemang Sylvester / American Journal of Engineering and Applied Sciences 2020, 13 (4): 803.818 DOI: 10.3844/ajeassp.2020.803.818

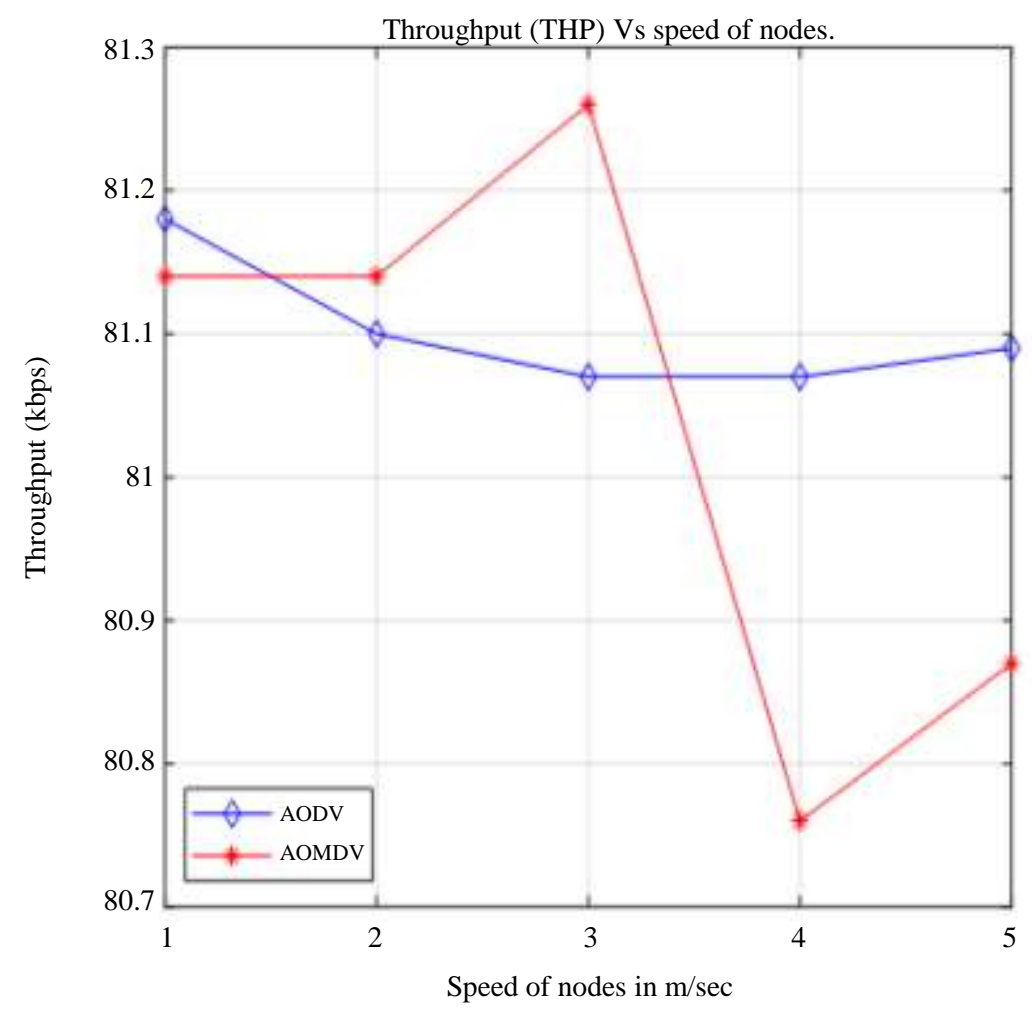

Fig. 7: Throughput with speed of nodes

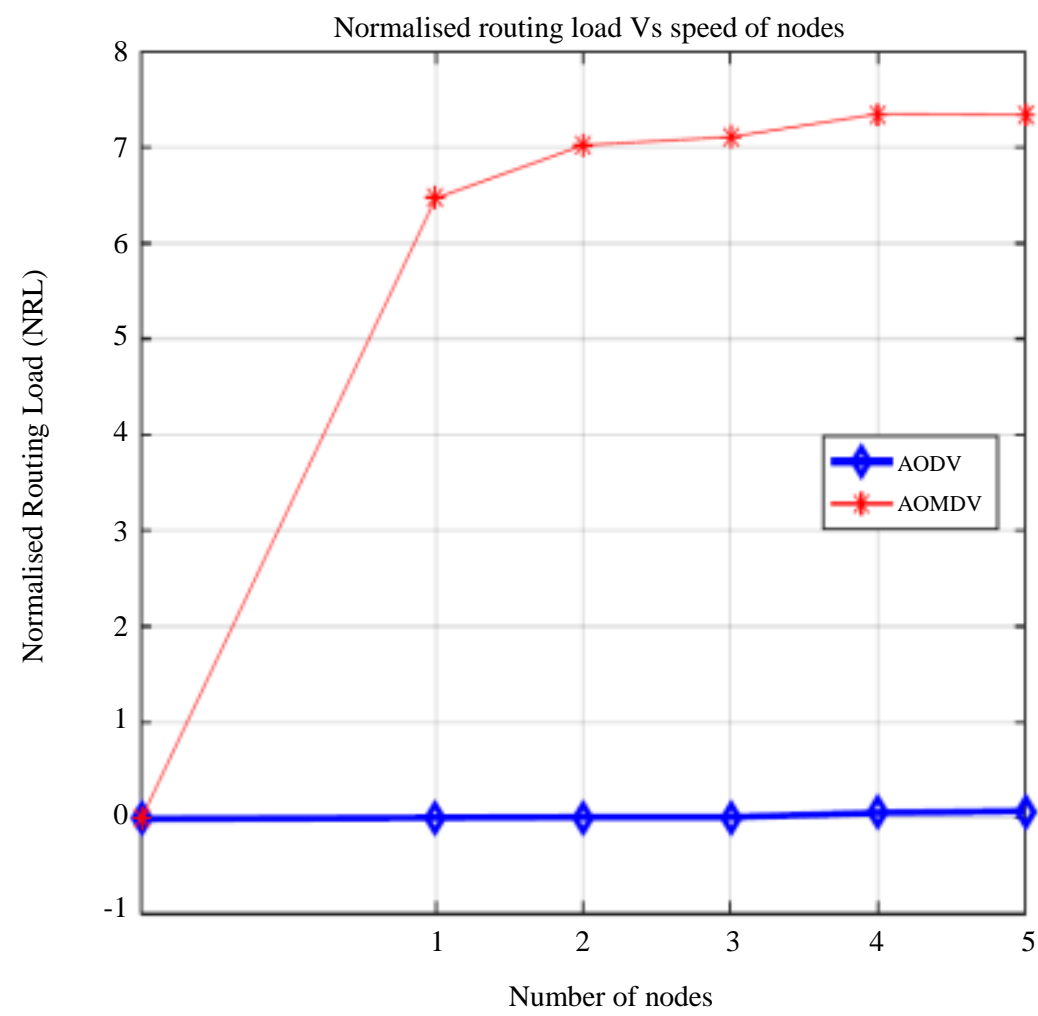

Fig. 8: Normalised routing load with speed of nodes 
Also, Fig. 8 presents NRL of AODV and AOMDV routing protocols with the increasing speed of nodes. It can be observed that the NRL of AODV outperformed that of AOMDV with the increasing speed of nodes. The NRL values of AODV routing are very negligible at all conditions, though they are increasing with increasing speed, whereas the NRL values of AOMDV not only grow but consist of higher values due to the used of multiple paths. The various paths are set up by AOMDV routing protocol through a lot of routing energy. This may due to nodes moving within each other's communication range, resulting in a faster path finding.

Again, when there are path disconnections and no alternative paths are available immediately, the protocol starts another path finding which contributes to the higher routing load in the network. Though AOMDV can respond somehow quicker at a cyclist's speed of 5 $\mathrm{m} / \mathrm{s}$ than AODV resulting in NRL decreasing but still supporting its higher values. Speeds increase can spike paths break more often.

Then, the need to commence new path findings and care. Consequently, the rate of path findings and maintenance is usually connected to the number of path breaks, thus causing NRL to increase.

Finally, Fig. 9 shows the AED of AODV and AOMDV routing protocols with the increasing speed of nodes. AODV has lower delay compared to that of AOMDV at nodes speeds of 1 to $3 \mathrm{~m} / \mathrm{s}$. AODV, however, has a higher delay at nodes speeds of 4 to 5 $\mathrm{m} / \mathrm{s}$. This is due to the single path discovery by AODV, in which it tries to reinitiate new or local path search resulting in lower AED except at a speed of 4 to $5 \mathrm{~m} / \mathrm{s}$ with increasing delay.

AOMDV, however, tries to find an alternative path to the destination from the multiple backup paths that result in a higher delay for the early period; thus, without immediately reinitiating path discovery. However, AED of AOMDV also increased at a speed of $5 \mathrm{~m} / \mathrm{s}$; it fell short of that of the AODV.

At such a high-speed AODV is unable to find and maintain path within the shortest possible time since link disconnections are very frequent and there are no alternative or backup paths to select from while AOMDV can choose a path from the backup paths for speedy packet delivery.

\section{Effect of Varying Number of Connections}

Figure 10 shows the PDR of AODV and AOMDV routing protocols with the number of connections between nodes. The PDR degraded as the number of connections increase. It can be observed that both routing protocols have similar PDR trend and are very close as the number of connections increases, with the PDR of AODV routing protocol slightly better than AOMDV protocol. This is because the number of connections increases leads to a rise in the number of communication nodes between sources - destinations pairs, which most of the times cause interferences and collisions. However, AODV can respond quickly to these link failures through its single path route discoveries and local search whereas AOMDV sometimes takes longer to react to such link disconnections due to its inability to always find an alternative path in the current backup paths. Therefore, there is a need to start a new path discovery all over again, leading to packet delivery slightly below that of AODV. Hence, when the number of connections is about half of the number of nodes may have dire consequences on the network.

Figure 11 shows the THP of AODV and AOMDV routing protocols with the increasing number of connections. It can be seen that both routing protocols THP are increasing almost linearly with the increased number of connections. Thus, the protocols can respond quickly to the increase in the number of connections leading to a consistent increase in THP. It can be noted that both routing protocols have very close THP with that of AODV slightly better than that AOMDV as the number of connections increase. This is as a result of the protocol quicker responds to increases in the number of nodes between sources - destinations pairs. The sending nodes in AODV either retransmit RREQ messages to rediscover or locally repair paths to reduce the congestions, collisions and interferences very quickly using a single path process.

Also, the AOMDV uses the multiple paths approach to opt out for an alternative path from the list of backup paths being maintained, resulting in an increased in THP in both AODV and AOMDV.

Furthermore, Fig. 12 presents NRL of AODV and AOMDV with the increasing number of connections. It can be seen that AOMDV has higher routing overhead than that of AODV for varying number of connections. This is due to the different approach between AODV and AOMDV routing protocols, which are based on single and multiple paths, respectively, even though they are both reactive protocols. Hence, AOMDV experiences more routing overhead than the AODV routing protocol. Though NRL of AOMDV decays as the number of connection increases, it is still higher than that of AODV.

Despite the low NRL values of AODV, there is a need for enhancement since there are some indications of possible increases with the increasing number of connections. 
John Tengviel and Agyemang Sylvester / American Journal of Engineering and Applied Sciences 2020, 13 (4): 803.818 DOI: 10.3844/ajeassp.2020.803.818

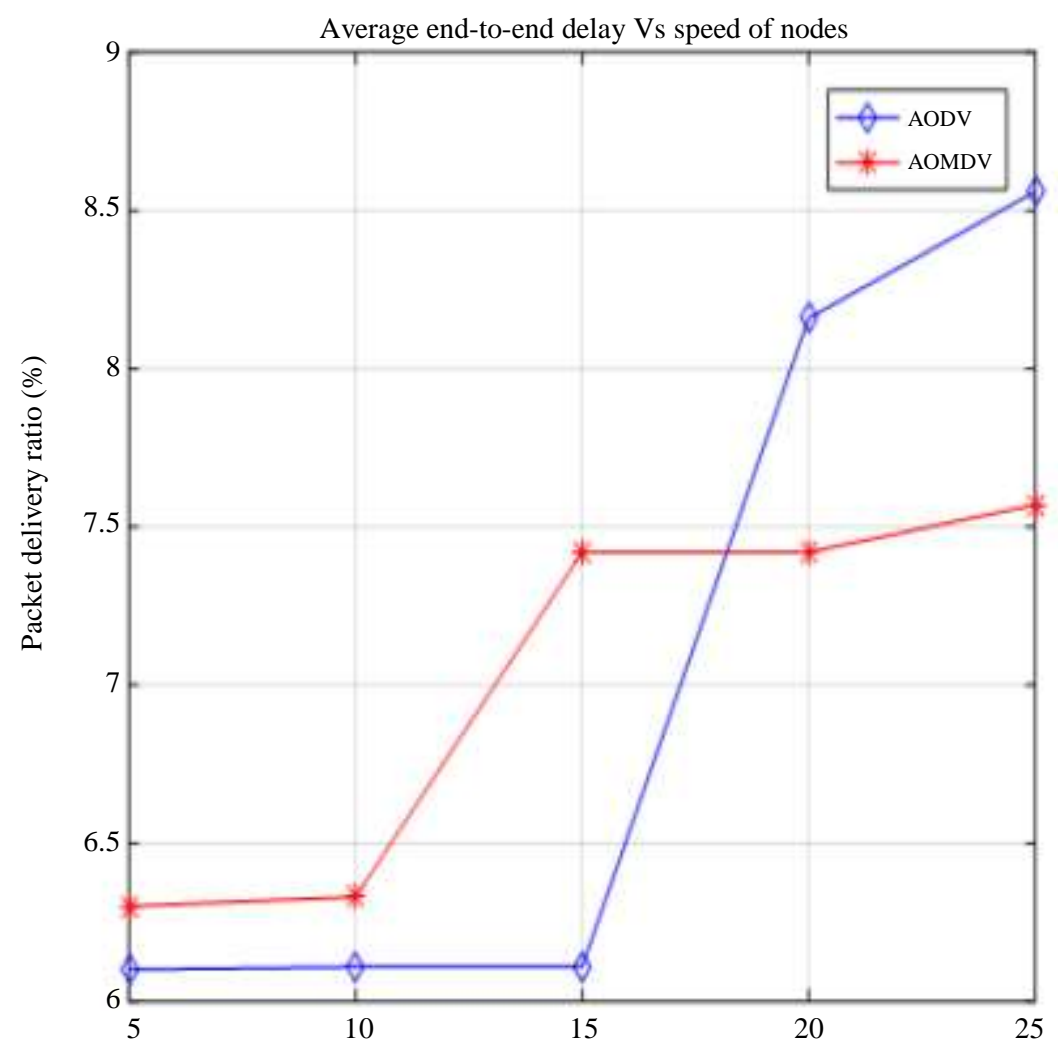

Fig. 9: Average end-to-end delay with speed of nodes

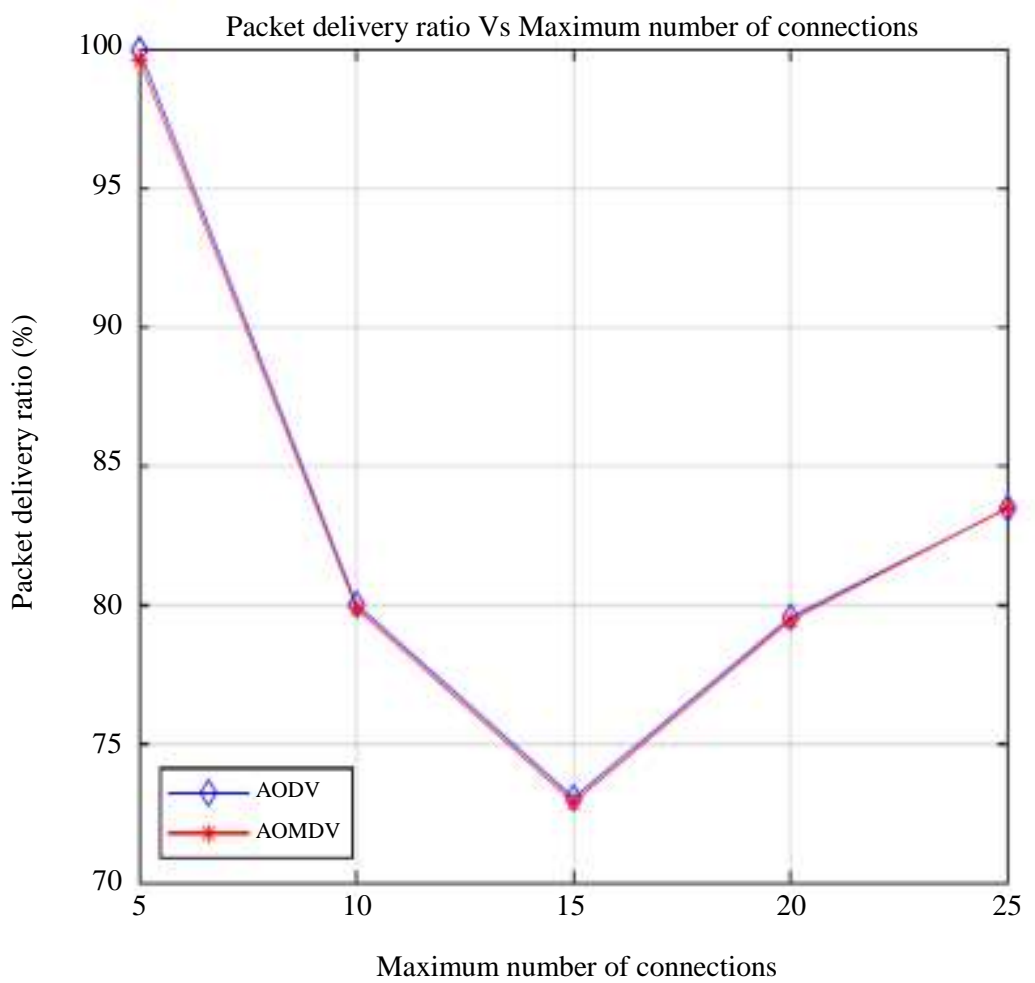

Fig. 10: Packet delivery ratio with number of connections 
John Tengviel and Agyemang Sylvester / American Journal of Engineering and Applied Sciences 2020, 13 (4): 803.818 DOI: 10.3844/ajeassp.2020.803.818

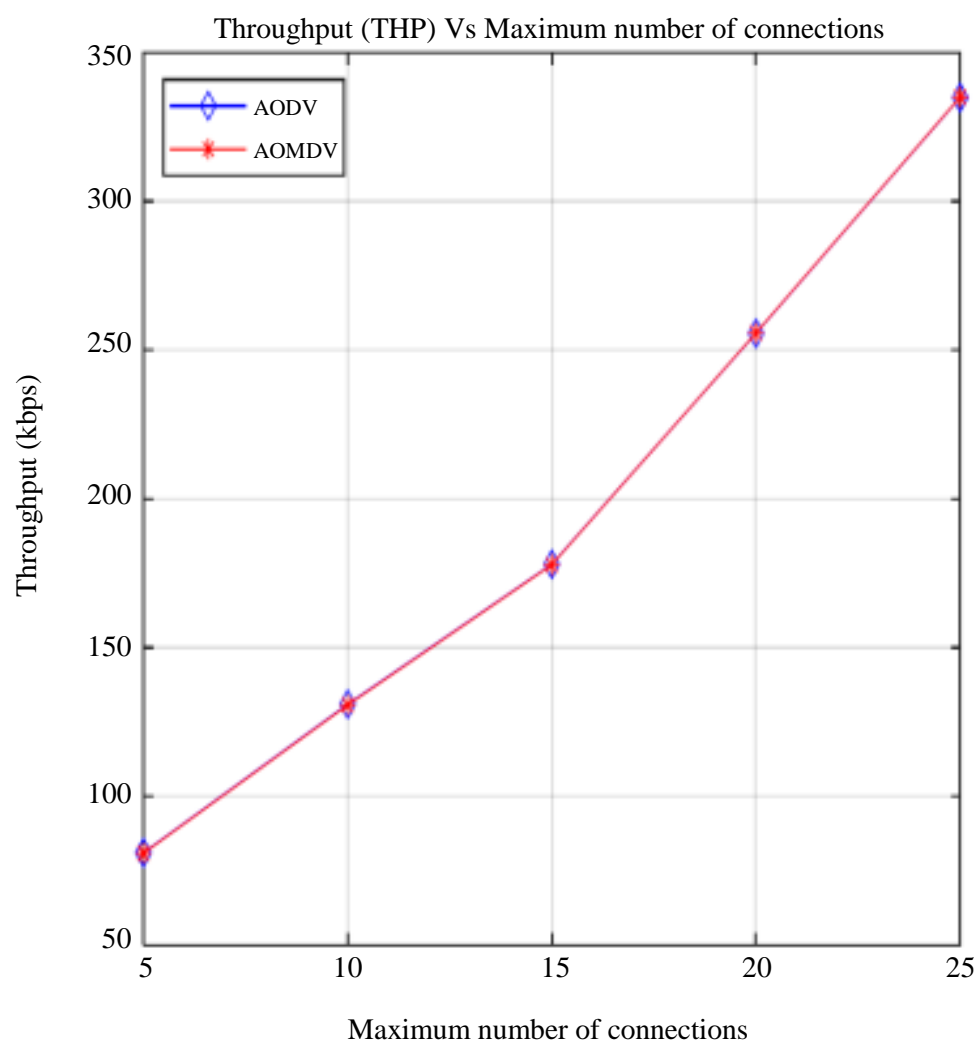

Fig. 11: Throughput with number of connections

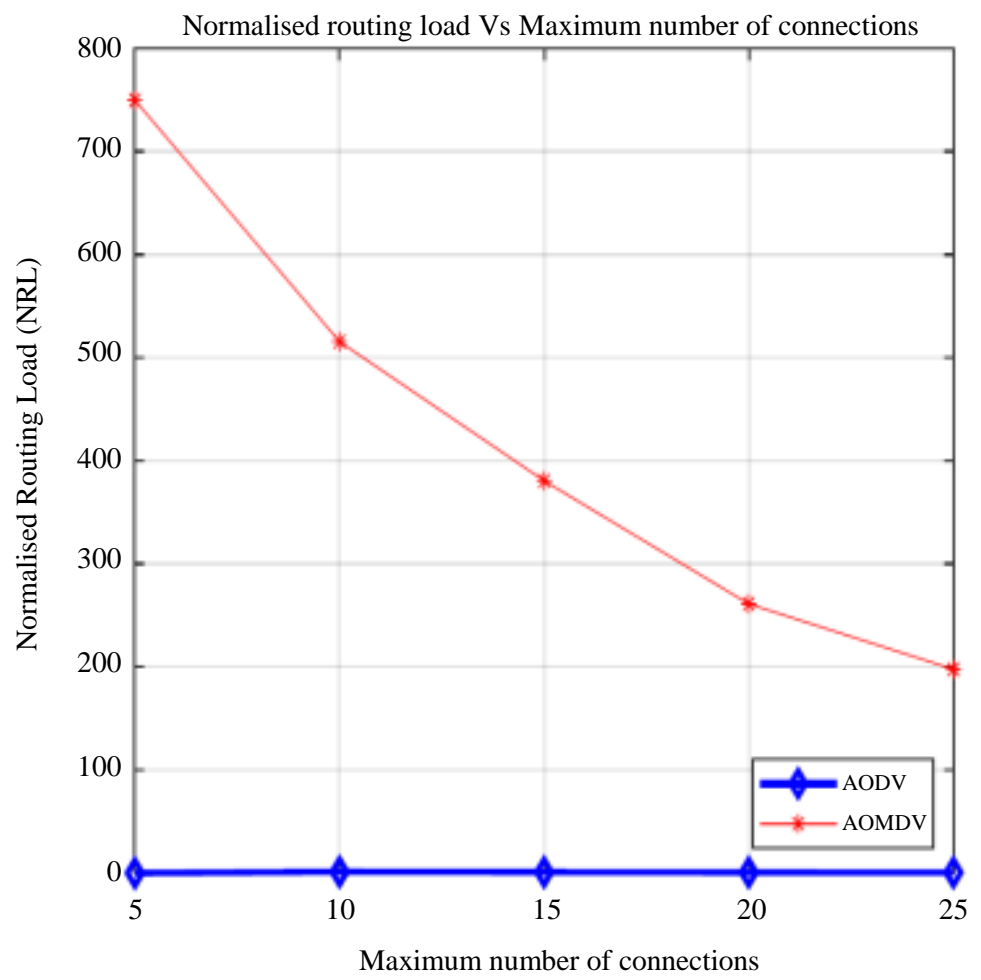

Fig. 12: Normalised routing load with number of connections 


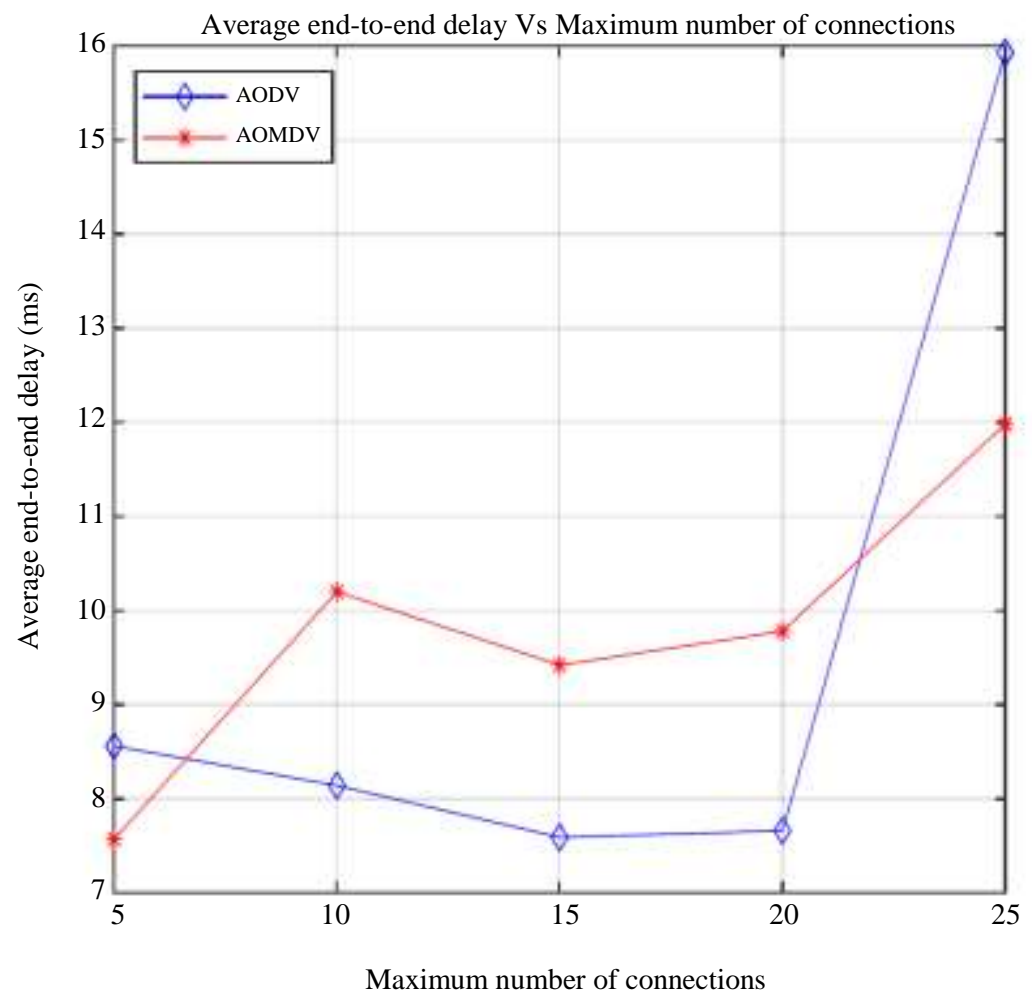

Fig. 13: Average end-to-end delay with the number of connections

Figure 13 presents AED of AODV and AOMDV routing protocols with an increased number of connections. It can be observed that AODV has a better AED than AOMDV in most cases except at 5 and 25 number of connections. This is because when a path disconnection happens in the network, AOMDV tries to find an alternative path from the multiple paths between the source and destination node pairs resulting in an additional delay to the packet delivery period.

Thus, as the number of sources - destinations pairs increase, different pairs of nodes attempt to broadcast to produce multipath resulting in congestions, interferences and collisions causing delays in forming, choosing of alternative paths and the sending of packets. The AED of AODV has low values as a result of fewer interferences and collisions due to clusters. However, there is still some level of packets delay when the number of connections increased. Hence the need to enhance the AODV routing protocol with a more realistic model.

\section{Conclusion and Future Works}

This study examines the performance of AODV and AOMDV routing protocols with a more realistic mobility model. The performance metrics are PDR, NRL, THP and AED with different scenarios such as the number of nodes, the speed of nodes and number of connections. It has been observed that the AODV routing protocol produced a better result than that of AOMDV. Thus, the AODV routing protocol performed better under a realistic mobility model.

The results presented in this study shows the significance of researchers to carefully investigate and implement routing protocols in MANETs in order to choose an appropriate protocol for enhancement since there are no definite observations that AOMDV outperforms AODV in all cases as reported in the related work. It also indicates that the choice of the mobility model has a significant impact on the routing protocol as stated in the literature in (Pandey and Mishra, 2017; Gupta et al., 2013).

In the future, we plan to enhance the AODV protocol and compare the enhanced routing with the AODV and AOMDV using the SMOOTH mobility model, as it is the most realistic mobility model that is readily available (Munjal et al., 2010; Bhandari et al., 2010; Pazand and McDonald, 2007; San San Naing and Tun, 2014).

\section{Acknowledgement}

This research study was conducted in Sunyani Technical University. The research received no specific grant from any funding agency in the public, commercial, or nonprofit organisations. 


\section{Author's Contributions}

All authors equally contributed to this manuscript.

\section{Ethics}

This article is precisely original and contains unpublished material. The corresponding author ensures that all of the other authors have read and approved the article and there are no ethical issues concerned.

\section{References}

Al Amri, H., Abolhasan, M., \& Wysocki, T. (2010). Scalability of MANET routing protocols for heterogeneous and homogenous networks. Computers \& Electrical Engineering, 36(4), 752-765.

Alslaim, M. N., Alaqel, H. A., \& Zaghloul, S. S. (2014, April). A comparative study of MANET routing protocols. In The Third International Conference on e-Technologies and Networks for Development (ICeND2014) (pp. 178-182). IEEE.

Bhandari, S. R., Lee, G. M., \& Crespi, N. (2010, May). Mobility model for user's realistic behavior in mobile ad hoc network. In 2010 8th Annual Communication Networks and Services Research Conference (pp. 102-107). IEEE.

Biradar, S. R., Majumder, K., Sarkar, S. K., \& Puttamadappa, C. (2010). Performance Evaluation and Comparison of AODV and AOMDV. International Journal on Computer Science and Engineering, 2(2), 373-377.

BonnMotion. (2016). A Mobility Scenario Generation and Analysis Tool, Documentation. BonnMotion. https://sys.cs.uos.de/bonnmotion/doc/README.pdf

Camp, T., Boleng, J., \& Davies, V. (2002). A survey of mobility models for ad hoc network research. Wireless communications and mobile computing, 2(5), 483-502.

Chen, B. R., \& Chang, C. H. (2003, July). Mobility impact on energy conservation of ad hoc routing protocols. In Proc. Int'l Conf. Advances in Infrastructure for Electronic Business, Education, Science, Medicine and Mobile Technologies on the Internet.

Chlamtac, I., Conti, M., \& Liu, J. J. N. (2003). Mobile ad hoc networking: imperatives and challenges. Ad hoc networks, 1(1), 13-64.

Ding, Y. M., Sun, C. H., Song, L., \& Kong, W. Q. (2012). Performance analysis of AODV protocol in mobile Ad Hoc networks. In Advanced Materials Research (Vol. 403, pp. 2415-2419). Trans Tech Publications Ltd.

Gupta, A. K., Sadawarti, H., \& Verma, A. K. (2013). Performance analysis of MANET routing protocols in different mobility models. International Journal of Information Technology and Computer Science (IJITCS), 5(6), 73-82.
Kevin, F., \& Kannan, V. (2011). The ns Manual. http://www.isi.edu/nsnam/ns/doc/ns_doc.pdf

Kocev, T., Mitrevski, P., \& Dimovski, T. (2013). Mobile Ad Hoc Communication-the Ability of Networking without Network.

Kumar, M., \& Mishra, R. (2012). An overview of MANET: History, challenges and applications. Indian Journal of Computer Science and Engineering (IJCSE), 3(1), 121-125.

Kumar, S., \& Kumar, S. (2015). Study of MANET: Characteristics, Challenges, Application, Routing Protocol and Security Attacks. International Journal of R\&D in Engineering, Science and Management, 2(5), 266-274.

Moudgil, S., \& Rana, S. (2017). Performance Investigation of Single Path and Multi Path Routing Protocols in High Mobility MANETs. International Journal of Computer Applications, 166(12).

Munjal, A., Camp, T., \& Navidi, W. C. (2010). SMOOTH: a simple way to model human walks. ACM SIGMOBILE Mobile Computing and Communications Review, 14(4), 34-36.

Munjal, A., Camp, T., \& Navidi, W. C. (2011, October). SMOOTH: a simple way to model human mobility. In Proceedings of the 14th ACM international conference on Modeling, analysis and simulation of wireless and mobile systems (pp. 351-360).

Obiniyi, A. A., \& Olanrewaju, O. M. (2015). Review of Mobile Ad Hoc Network Protocols.

Ofosu, K. K., Abdulai, J. D., \& Katsriku, F. A. (2018a). Performance evaluation of Mobile IP on mobile ad hoc networks using NS2. Computer Science \& Information Technology (CS \& IT), 15-27.

Ofosu, K. K., Abdulai, J. D., \& Katsriku, F. A. (2018b). Mobile IP on Mobile Ad Hoc Networks: An Implementation and Performance Evaluation Using NS2. Advanced Computing: An International Journal (ACIJ), 9(2).

Pandey, M. R., \& Mishra, R. K. (2017). The literature review: performance enhancement of mobility models in wireless ad hoc network. International Journal of Modern Trends in Engineering and Research. 4(4), 10.

Parekh, V. A. (2013). Performance Evaluation of AODV and AOMDV Routing Protocols in MANET. International Journal of Advanced Research in Computer Science, 4(3).

Parita, O., \& Pooja, S. (2014). Performance Evaluation of Ad-Hoc on-Demand Distance Vector Routing Protocol and its Multi-Path Variant AOMDV. International Journal of Computer Science \& Communication. 5(1): p. 3.

Patil, V. P. (2012). Impact of Mobility and Network load on the Performance of Reactive and Proactive Routing Protocol in Manet. IJCES (International Journal of Computer Engineering and Science), 2, 8-16. 
Patil, V. P., Patil, K. T., Kharade, A. R., \& Gote, D. (2012). Performance enhancement of reactive on demand routing protocol in wireless Ad Hoc network. International Journal of Smart Sensors and Ad Hoc Networks (IJSSAN), 1(4).

Pazand, B., \& McDonald, C. (2007, July). A critique of mobility models for wireless network simulation. In 6th IEEE/ACIS International Conference on Computer and Information Science (ICIS 2007) (pp. 141-146). IEEE.

Prabhu, M. A., \& Krishnan, M. S. (2015). Comparison of AODV and AOMDV routing protocols.

San San Naing, Z. M. N., \& Tun, H. M. (2014). Performance Of Routing Protocols For Mobile Ad Hoc Networks.

Sedrati, M., Bilami, A., \& Benmohamed, M. (2011). MAODV: AODV variant to Improve Quality of Service in MANETs. arXiv preprint arXiv: 1104.1186.

Shafi, N. A. I., \& Abidi, S. (2013). Evaluation and Performance of Reactive Protocols Using Mobility Model. arXiv preprint arXiv:1310.5848.

Singh, B., Baghla, S., \& Monga, H. (2017). Mobility models based performance evaluation of AOMDV routing protocol of MANET. Int. J. Appl. Res, 3, 82-86.

Soni, R., Saxena, A., \& Shrivasta, A. (2013). The Efficient AODV routing protocol in MANET. International Journal of Application or Innovation in Engineering \& Management, 2(6).
Tengviel, J., \& Diawuo, K. (2013). Comparing the Impact of Mobile Nodes Arrival Patterns in Manets Using Poisson and Pareto Models.

Tengviel, J., Diawuo, K., \& Dotche, K. A. (2012). The effect of the number of mobile nodes on varying speeds of manets. arXiv preprint arXiv:1212.2567.

Tengviel, J., K. Dotche and K. Diawuo, (2013). The impact of mobile nodes arrival patterns in mobile ad hoc networks using poisson models. International Journal of Managing Information Technology. 4, 55-71.

Ubuntu-14.04. (2016). Alternative downloads. Ubuntu. http://www.ubuntu.com/download/alternativedownloads

Varshney, A., \& Maheshwari, P. (2016). Comparative Study of AODV \& AOMDV Routing Protocol. Int J Control Theory Appl, 9(6).

Vijayavani, G. R., \& Prema, G. (2012, August). Performance comparison of MANET routing protocols with mobility model derived based on realistic mobility pattern of mobile nodes. In 2012 IEEE International Conference on Advanced Communication Control and Computing Technologies (ICACCCT) (pp. 32-36). IEEE.

VirtualBox. (2016). VirtualBox-5.0.26-108824-Win updated in July, https://www.virtualbox.org/wiki/Download_Old_Bu ilds_5_0 